\title{
Myofibroblastic Inflammatory Bowel Tumor: Unusual location, Case Report
}

\author{
Khalid Gharbi, Mohammed Amine Lkousse, Jamal Atmani, Younes Ismail, Abdeltif Elfarouki, \\ Adil Ait Errami, Sofia Oubaha, Zohor Samlani, Khadija Krati
}

Gastroenterology Service, Hospital Mohammed VI, Marrakech, Morocco

Email address:

khalidgharbi@gmail.com (K. Gharbi)

\section{To cite this article:}

Khalid Gharbi, Mohammed Amine Lkousse, Jamal Atmani, Younes Ismail, Abdeltif Elfarouki, Adil Ait Errami, Sofia Oubaha, Zohor Samlani, Khadija Krati. Myofibroblastic Inflammatory Bowel Tumor: Unusual location, Case Report. International Journal of Gastroenterology.

Vol. 6, No. 2, 2020, pp. 25-27. doi: 10.11648/j.ijcems.20200602.12

Received: January 15, 2020; Accepted: February 10, 2020; Published: June 4, 2020

\begin{abstract}
Inflammatory myofibroblastic tumours (IMT) are unusual benign tumours, also known as inflammatory pseudotumours, represent a group of fibro-inflammatory lesions of recent individualization. They are mesenchymal tumours of intermediate class, very rare and of unknown etiology. They are initially described in the lungs, but have been observed in several other locations, mainly abdominal and pelvic. Its location in the colon is exceptional. The evolutionary doubt of these tumours requires regular and long term follow-up. We report the case of a 30-year-old patient who presented with a stenosing caecal mass. After surgical removal, histological and immunohistochemical examination was in favor of a colonic (IMT). The clinical, radiological and histological characteristics of this anatomoclinical entity will be discussed in light of the data in the literature.
\end{abstract}

Keywords: Inflammatory Myofibroblastic Tumor, Pseudotumor, Benign, Immunohistochemistry

\section{Introduction}

Inflammatory myofibroblastic tumours (IMT), also known as inflammatory pseudotumours, represent a group of fibro-inflammatory lesions of recent individualization. They are mesenchymal tumours of intermediate class, very rare and of unknown etiology. Initially described in the lung they have been reported in most organs and anatomical sites [1-3]. Their localization in the colon is extremely rare. We report the observation of a 30 -year-old patient with colonic (IMT).

\section{Case Report}

The patient was 30 years old, with no particular history and had been presenting for four months with pain in the right iliac fossa without any notion of vomiting or digestive haemorrhages (haematemesis, melena). On admission, he was apyretic, the abdominal examination found a palpable mass in the right iliac fossa which was not well limited, fixed and slightly painful, without hepatosplenomegaly, without ascites, without palpable adenopathy and the rectal examination was normal, all of which evolved in a preserved general state.

Biologically assessment, showed normocytic normochromic anemia at $9.5 \mathrm{~g} / \mathrm{dl}$, white blood cell count $7300 / \mathrm{mm} 3$, platelet count $354,000 / \mathrm{mm}^{3}$, C-reactive protein $104 \mathrm{mg} / \mathrm{L}$, accelerated sedimentation rate to $62 \mathrm{~mm}$ in the first hour, and tumour markers CA 19-9 and ACE were normal.

Abdominal CT scan showed a stenosing caecal solid mass of $6 \times 5 \times 4 \mathrm{~cm}$ of heterogeneous non-metastatic density, without peritoneal effusion. Colonoscopy revealed a stenosing caecal mass and a right hemicolectomy removing the mass by median laparotomy was performed with anterolateral ileocolic anastomosis.

On an anatomopathological examination of the surgical specimen, the tumour is composed of cells resembling fibroblasts and spindle-shaped myofibroblasts with elongated hyperchromic nuclei with a mitotic index estimated at less than 2 mitoses per 10 fields at high magnification. The cytoplasm is sparsely eosinophilic. The stroma is loose fibrous, richly vascularized, with a dense inflammatory infiltrate of lymphocytes, plasma cells and eosinophilic polynuclear cells organized in numerous lymphoid follicles, which proliferates submucosally and extends to the muscularis. (Figure 1). Surgical limits are 
healthy. Node removal has brought back 7 nodes which are the site of reactive lymphadenitis lesions.
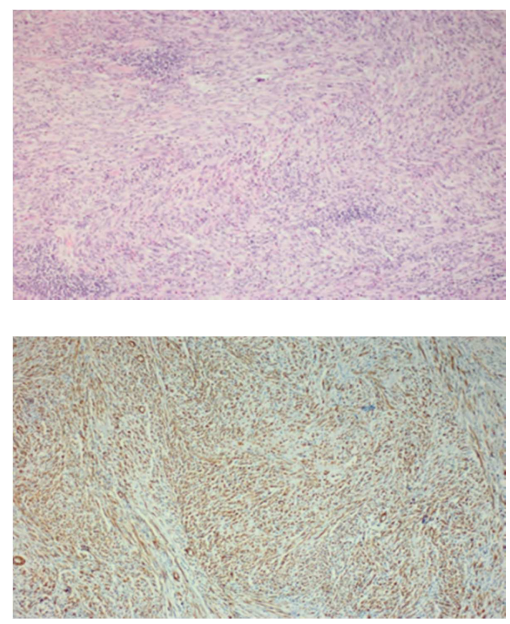

Figure 1. Fusocellular proliferation within a hyaline stroma associated with Inflammatory cells (lymphocytes, plasma cells and macrophages).

Immunohistochemistry was positive for actin, desmin and negative for CD117, S-100, pancytokeratin, bcl-2, CD34, and anaplastic lymphoma kinase (ALK), and the final diagnosis was colonic (IMT).

After surgery, the patient improved and is scheduled for follow-up to detect tumor recurrence.

\section{Discussion}

IMT were first described by Brunn in 1937 as a primary lung tumour, consisting of a proliferation of fibroblastic and myofibroblastic cell infiltrates [1]; however, multiple extrapulmonary manifestations have been reported $[2,3]$. Extrapulmonary sites may include the upper respiratory tract, mesentery, genitourinary tract, gastrointestinal tract, mediastinum, retroperitoneum, pelvis, trunk, extremities, head, neck, spleen, brain, pancreas and liver [3], the digestive localization was first described by Vanek in 1949 in the stomach [4], the colonic localization remains exceptional [4].

The etiological factors responsible for the development of IMT are not clearly established. Some researchers believe it is a true neoplasm, while others believe it represents an immunological response to an infectious or non-infectious agent [5].

Clinical signs are varied, and include abdominal pain, meteorism, nausea, vomiting, diarrhea or constipation. Compressive phenomena due to the progressive increase in tumour size may be seen. In rare cases, significant weight loss and stigma of digestive haemorrhage are observed [6].

At the level of the appendix, there are: An acute pseudo appendicular form, sometimes atypical abdominal pain, febrile occlusions or profuse diarrhoea. Biological explorations show an inflammatory syndrome, but tumour markers are negative (ACE, CA19-9) [7].

Radiologic semiology is poorly defined due to the polymorphism of the (IMT): A single large lesion is usually found, exerting a mass effect on neighboring organs. Its margins are more or less well limited. The CT scan reveals a lesion that is hypodense or isodense in relation to the muscles, homogeneous or heterogeneous. Calcifications and adenomegalia are rare. Contrast is variable [8]: contrast is absent, early or late; homogeneous or not. The same semiological elements are found in MRI.

The delay in contrast taking depends on its vascular quota: early contrast taking in the case of young fibrosis, cellular inflammatory and oedematous; late contrast taking if the fibrosis is mature, collagen. It is therefore theoretically desirable to carry out late acquisitions, in order to highlight late contrast acquisition, even discreetly.

The macroscopic appearance of a (IMT) is a single, polylobed, unencapsulated, firm mass with a yellow or brownish surface, destroying the slowly growing tissue it invades. It may have necroticohemorrhagic changes and calcifications. The size ranges from 1 to $20 \mathrm{~cm}$ with an average of $7 \mathrm{~cm}$ [9]. Microscopically, there is a component of spindle-shaped cells of myofibroblastic type [10], more or less fasciculated, in a hyaline stroma, associated with numerous lympho-plasmocytic inflammatory cells. There is little mitotic activity.

For the final diagnosis of IMT, immunohistochemical analysis is very important. Tumor cells are typically actin-positive with or without desmin expression and do not express CD117 and CD34, S100 [11]. In our case, the tumor cells were actin-positive, negative for S100, ALK, and there was no expression of CD117, CD34. [12]

Differential diagnoses include non-tumourous lesions, such as abscesses or tuberculous granulomas, and benign or malignant tumour lesions. Lymphoma or GIST should also be ruled out.

Finally, the diagnostic and therapeutic means remains the surgical removal of the tumour. Indeed, the lack of specificity of the preoperative examinations and the unreliability of the colonic biopsy limit the possibilities of a pre- or intraoperative diagnosis. Carcinologic excision, when it is possible, is necessary and therefore the only way to ensure diagnostic certainty. [13].

One of the major problems of this pathology and determining its prognosis [14]. At present, no factor can predict the evolution of inflammatory pseudotumours, which is often benign, but malignant transformation is possible. The prognosis is generally good after removal of the tumour and recurrences are rare [13].

\section{Conclusion}

Inflammatory pseudotumours of the colon are exceptional, the clinical and radiological manifestations can be those of a malignant tumour. The diagnosis of certainty is based on the surgical excision which allows the histological study. The evolutionary doubt of these tumours requires regular and long term follow-up. 


\section{References}

[1] Coffin C M, Watterson J, Priest J R, Dehner L P. Extrapulmonary inflammatory myofibroblastic tumor (inflammatory pseudotumor). A clinicopathologic and immunohistochemical study of 84 cases. Am J Surg Pathol. 1995; 19 (8): 859-872. [PubMed] [Google Scholar].

[2] Kim E Y, Lee I K, Lee Y S. et al. Inflammatory myofibroblastic tumor in colon. J Korean Surg Soc. 2012; 82 (1): 45-49. [PMC free article] [PubMed] [Google Scholar].

[3] Narla L D, Newman B, Spottswood S S, Narla S, Kolli R. Inflammatory pseudotumor. Radiographics. 2003; 23 (3): 719 729. [PubMed] [Google Scholar].

[4] Karnak I, Senocak M E, Ciftci A O. et al. Inflammatory myofibroblastic tumor in children: diagnosis and treatment. J Pediatr Surg. 2001; 36 (6): 908-912. [PubMed] [Google Scholar].

[5] Hedlund G L, Navoy J F, Galliani C A, Johnson W H Jr. Aggressive manifestations of inflammatory pulmonary pseudotumor in children. Pediatr Radiol. 1999; 29 (2): 112116. [PubMed] [Google Scholar].

[6] Benjamin SP, Hawk WA, Turnbull RB. Fibrous inflammatory polyps of the ileum and cecum review of five cases with emphasisou differentiation from mesenchymal neoplasm. Cancer 1977; 39: 130-5.

[7] Jougon J, Amar AR. Pseudotumeur inflammatoire de l'appendice à propos d'un cas et revue de la littérature. J Chir 1991; 128: 86-8.

[8] Levy A. D., Shaw J. C., Sobin L. H. Secondary tumors and tumorlike lesions of the peritoneal cavity: imaging features with pathologic correlation Radiographics 2009; 29 (2): 347-373.

[9] Gleason B. C., Hornick J. L. Inflammatory myofibroblastic tumours: where are we now? J Clin Pathol 2008; 61 (4): 428-437.

[10] Coffin cm, Patel A., Perkins S., Elenitoba-Johnson K. S., Perlman E., Griffin C. A. ALK1 and p80 expression and chromosomal rearrangements involving 2 p23 in inflammatory myofibroblastic tumor Mod Pathol 2001; 14 (6): 569-576.

[11] Salameh M, Sultan I, Barbar M. et al. Inflammatory myofibroblastic tumor causing unexplained anemia in a toddler: a case report. J Med Case Reports. 2011; 5: 69. [PMC free article] [PubMed] [Google Scholar].

[12] Miettinen M, Sobin L H, Sarlomo-Rikala M. Immunohistochemical spectrum of GISTs at different sites and their differential diagnosis with a reference to CD117 (KIT) Mod Pathol. 2000; 13 (10): 1134-1142. [PubMed] [Google Scholar].

[13] Maiga MY. Pseudotumeur inflammatoire du foie: à propos d'un cas. Annales de Chirurgie 2003; 128: 273-4.

[14] Coffin CM, Watterson J, Priest J R, Dehner LP. Extrapulmonary inflammatory myofibroblastique tumor (inflammatory pseudotumor). A clinicopathologic and immunohistochimical study of 84 cases. Am J of Surg Pathol 1995; 19: 859-72. 